Introduction 



\title{
25 YEARS OF PAH HYPOTHESIS
}

\author{
A.G.G.M. Tielens ${ }^{1}$
}

\begin{abstract}
Strong IR emission features at 3.3, 6.2, 7.7, 8.6, and $11.2 \mu \mathrm{m}$ are a common characteristic of the interstellar medium of the Milky Way and nearby galaxies and out to redshifts of 3 . Here, we review the history of the PAH hypothesis which attributes these emission features to vibrational fluorescence of large $(\sim 50$ carbon-atom Polycyclic Aromatic Hydrocarbon molecules pumped by ultraviolet photons from nearby stars or the average interstellar radiation field. Over the last 25 years, our insight in the characteristics of these molecules and their role in the Universe has greatly improved and the PAH hypothesis is alive and well; not in the least due a remarkable adaptability. Not surprisingly, the precise characteristics of these species remains to be defined.
\end{abstract}

\section{The early years}

Over the last three decades our understanding of the interstellar medium has increased dramatically. To a large extent this has been driven by rapid developments in infrared detector technology driven by the Cold War. Sensitive infrared spectrometers operating in all the infrared windows are now standard on all major ground-based infrared observatories and major infrared space missions have probed the Universe at all infrared wavelengths. As soon as the infrared sky opened up for spectroscopy in the early 70ies, Gillett et al. (1973) discovered broad emission features in the $\mathrm{L}(3 \mu \mathrm{m})$ and $\mathrm{N}(10 \mu \mathrm{m})$ band windows (Fig. 1). While a good fit could be obtained to the profile and peak position of the $11.3 \mu \mathrm{m}$ band with carbonates, air-borne observatories refuted this identification since the strong band at $6.85 \mu \mathrm{m}$ was not detected (Russell et al. 1977; Willner et al. 1979). Instead, these observations revealed that the the 3.3 and $11.3 \mu \mathrm{m}$ bands were part of a set of strong bands - together with bands at $6.2,7.7$, and $8.6 \mu \mathrm{m}$ - that occured together. These and subsequent observations established that these broad

${ }^{1}$ Leiden Observatory, Leiden University, PO Box 9513, Leiden, The Netherlands 


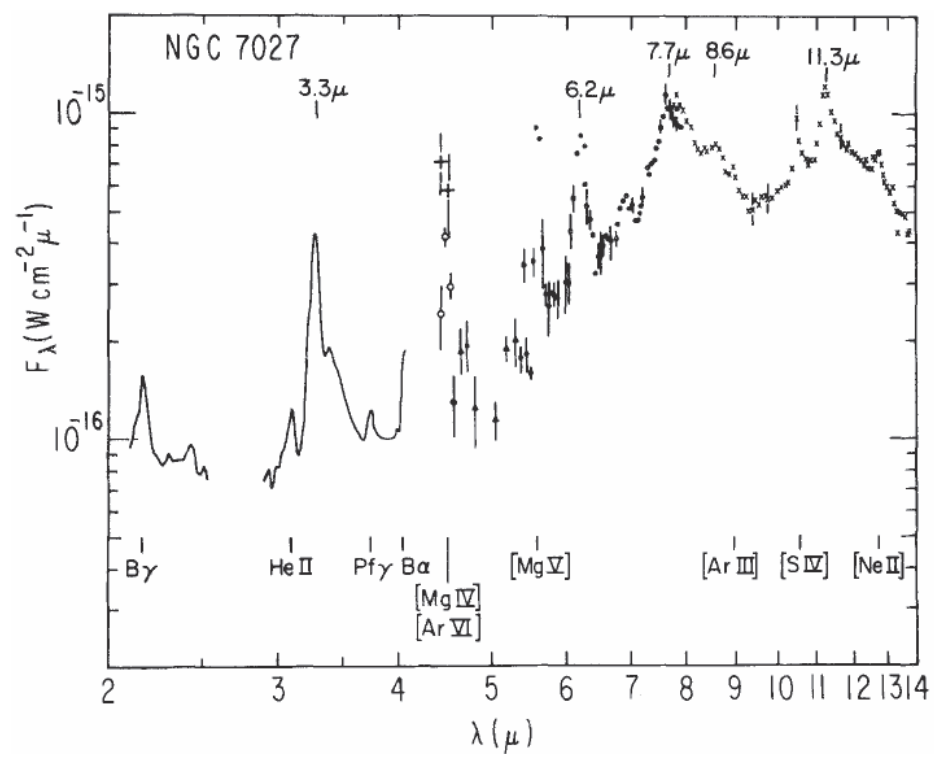

Fig. 1. The first overview spectrum of the PAH emission bands obtained from the ground in the $\mathrm{L}$ and $\mathrm{N}$ band windows and from the Kuiper Airborne Observatory $(5-8 \mu \mathrm{m})$. Taken from the review by Willner et al. (1979).

IR emission features were a common characteristic of all objects where strong ultraviolet (UV) photons illuminated nearby material. Surprisingly, the nature of the objects seemed irrelevant: planetary nebulae, HII regions, reflection nebulae, and starburst galaxies all showed the same set of emission features. For almost a decade, identification of the carrier remained mysterious and these bands were collectively called the Unidentified InfraRed (UIR) bands. In the early 80ies, Duley \& Williams (1981) realized that the peak positions of the UIR bands were very characteristic for aromatic materials and they proposed Hydrogenated Amorphous Carbon grains as the carriers. This proposal highlighted, however, an energetic issue inherent to all "bulk" grain approaches for the UIR bands: The emission originated far from the illuminating star where the radiative equilibrium temperature of grains is much too low $\left(T_{d} \sim 10-75 \mathrm{~K}\right)$ to account for emission in the nearand mid-IR range. The next - and key step - was provided by Kris Sellgren 1984 who studied the IR emission from reflection nebula where this "energy" crisis was particularly rampant. She realized that the limited heat capacity of very small grains would result in large temperature excursions upon absorption of a single UV photon. Assuming a typical UV photon energy of $10 \mathrm{eV}$, a size of $50 \mathrm{C}$-atoms was implied. Independently, Léger \& Puget (1984) and Allamandola et al. (1985) put these concepts together and the PAH hypothesis was born: the IR emission features at 3.3, 6.2, 7.7, and $11.3 \mu \mathrm{m}$ are carried by large Polycyclic Aromatic Hydrocarbon molecules containing some $50 \mathrm{C}$-atoms and with a drived abundance 
of $5-10 \%$ of the elemental carbon, these molecules - as a class - are the most abundant organic molecules in space.

In these early years, there was much discussion on the character of the carriers of the UIR bands and a plentitude of materials were proposed including PAH molecules, HAC grains, Quenched Carbonaceous Composite, and coal. At the Santa Clara conference at the end of the 80ies, the situation was summarized by the title of the session on the IR emission bands: "the Overidentified InfraRed bands" (Allamandola \& Tielens 1989). All of these materials shared however the basic molecular structure of PAH molecules: benzene rings fused together into aromatic structures decorated with hydrogen at the edge, although many of these materials also contained other molecular structures often with other elements besides $\mathrm{C}$ and $\mathrm{H}$. While the idea that the IR emission bands are carried by large molecules rather than grains is now undisputed, the exact structure of the species and in particular the presence of heteroatoms are still widely and vividly debated.

\section{The PAH hypothesis}

This heralded an exciting period were new ideas were announced at a rapid pace. In particular, at about the same time Kris Sellgren performed her breakthrough study, the IRAS mission discovered widespread emission at 12 and $25 \mu \mathrm{m}$ in the diffuse interstellar medium of the Milky Way and other galaxies (Low et al. 1984). While there was no spectrum, it was quickly surmised that this emission was, in essence, the UIR bands at $7.7,8.6$, and $11.3 \mu \mathrm{m}$ in combination with a continuum steeply rising towards longer wavelengths and all pumped by the interstellar radiation field (Puget et al. 1985). Hence, PAH molecules had to be ubiquitous throughout the interstellar medium. It was also realized that such molecules would also have absorption bands in the visible and UV range. In fact, the wellknown Diffuse Interstellar Bands (DIBs) in the visible region of the spectrum might well be due to ionized or "radical" members of the PAH family (van der Zwet \& Allamandola 1985; Crawford et al. 1985; Leger et al. 1985) and these bands may well hold important clues for the identification of individual interstellar PAHs. Because of their high abundance, it was also quickly clear that PAH molecules would have an enormous influence on the energy and ionization balance of interstellar gas both in the diffuse and dense media and hence on the phase structure, gas phase composition, and ambipolar diffusion of clouds (Verstraete et al. 1990; Lepp \& Dalgarno 1988). Many of these ideas are summarized in the first reviews (Leger \& Puget 1989; Allamandola et al. 1989). At this point, the PAH hypothesis can be summarized as:

- A substantial fraction of the carbon in the universe is in form of PAH molecules.

- A substantial fraction of the UV-visible radiative stellar energy in galaxies is "transformed" by PAHs to the mid-IR range. 


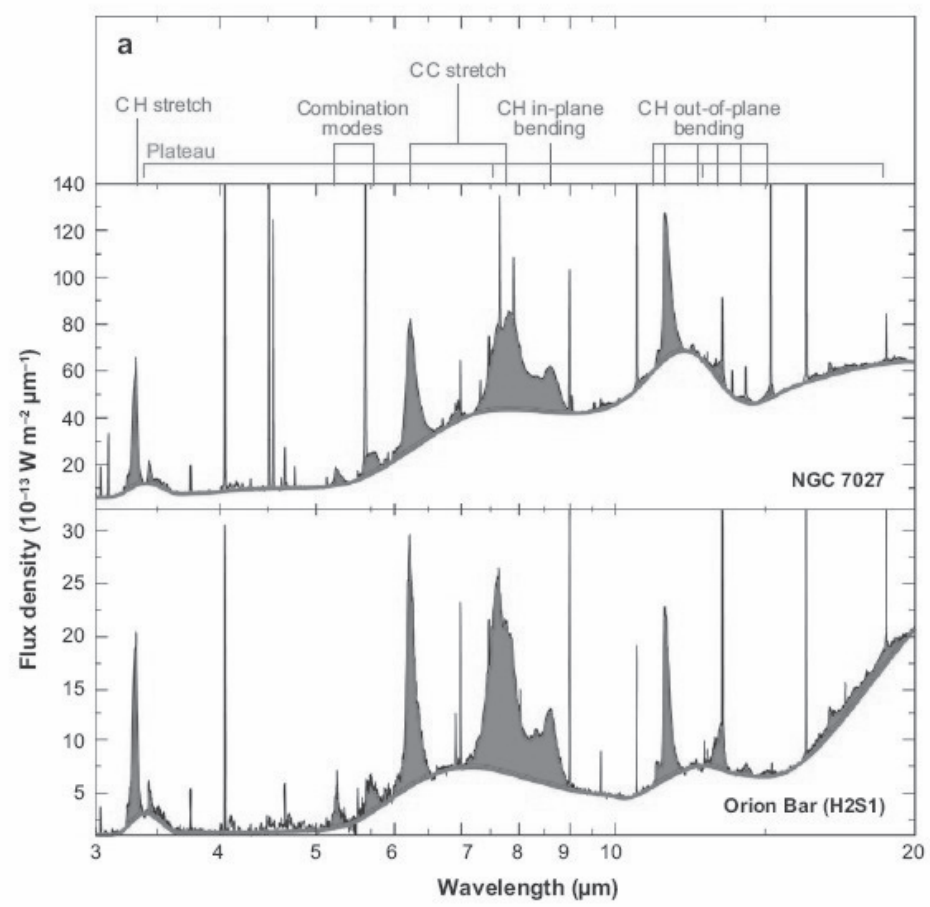

Fig. 2. The mid-infrared spectra of the PhotoDissociation Region in the Orion Bar and in the Planetary Nebulae, NGC 7027 are dominated by a rich set of emission features. Assignments of these features with vibrational modes of PAH molecules are labeled at the top. Figure adapted from Peeters et al. (2002).

- PAH molecules may provide observational signatures throughout the full spectral range from the extreme ultraviolet to the radio regime.

- PAHs are destroyed by hard UV radiation and thus they must be at the basis of a complex chemistry in the ISM.

- PAHs contribute to many key physical processes that affect the basic structure and evolution of the interstellar medium of galaxies.

\section{The space-based era}

The opening of the full infrared window by spectroscopic space missions further increased our knowledge of the interstellar PAHs. The Low Resolution Spectrometer (LRS) - a slitless spectrometer sensitive from 7.5 to $23 \mu \mathrm{m}$ with a resolving power of $R \sim 20$ - on the InfraRed Astronomy Satellite (IRAS) has provided a first systematic overview (Olnon 1986) of the extent of the UIR bands. The Short Wavelength Spectrometer, SWS (de Graauw et al. 1996) on board of the Infrared 
Space Observatory (ISO), represented the next big step forward by providing complete 2.5 to $45 \mu \mathrm{m}$ spectra of essentially all IR-luminous galactic sources with resolutions ranging from $\sim 200$ up to $\sim 2000$ (for the brightest sources). The ISO mission also carried the ISOCAM (Cesarsky et al. 1996) aboard - which in CVFmode provided spectral-imaging capabilities over $\sim 1$ square arcminute areas with pixel sizes of $3-6$ arcseconds at a modest spectral resolution of 50 - that produced equally important studies of interstellar PAHs. Together these instruments revealed the dominance of the UIR bands for essentially all bright sources that they could study. The InfraRed Spectrometer (IRS) on board of the Spitzer Space Telescope - operating from 5.2 to $38 \mu \mathrm{m}$ at low spectral resolution (60 to 130) and from 10 to $37 \mu \mathrm{m}$ at moderate resolution $(R \sim 600)$ but with greatly superior sensitivity over ISO (Houck et al. 2004) - allowed systematic spectroscopic studies of large samples of even weak sources in the Milky Way, nearby galaxies and in bright galactic nuclei out to redshifts of $\sim 3$. All of these studies together demonstrated that PAHs dominate the spectra of almost all objects, including HII regions, reflection nebulae, young stellar objects, Planetary Nebulae, postasymptotic-giant-branch objects, nuclei of galaxies, and ultraluminous infrared galaxies (ULIRGs). Moreover, the IR cirrus, the surfaces of dark clouds, and the general interstellar medium (ISM) of galaxies are set aglow in these IR emission features (for a recent review see Tielens 2005).

\section{PAHs in the far Universe}

The high sensitivity of the Spitzer instruments has demonstrated that PAH emission is present out to redshifts of $\mathrm{z} \simeq 3$. Regular and starburst galaxies in the near Universe are readily surveyed with Spitzer and for example the SINGS sample as well as open and guaranteed time programs are rife with galaxies whose spectra are dominated by the mid-IR emission features (e.g., Smith et al. 2007). With these studies, reliable criteria could be established that allow the use of the PAH emission strength as tracers of the star formation rate (see Calzetti, elsewhere in this volume). With IRS/Spitzer, galaxies much farther away could still be studied in the case of lens systems. The cloverleaf system at a redshift of 2.58 is a prime example (Fig. 3; Lutz et al. 2007) but many more studies exist (Rigby et al. 2008; Lutz et al. 2005). As a class, the ultraluminous sub-millimeter galaxies were also within reach out to redshifts of $\sim 3$ with Spitzer (Pope et al. 2008).

In a more indirect way, Spitzer probed distant but less luminous systems through source counts. The spectrum of a galaxy will shift further into the infrared as its distance increases. Because the PAH features dominate the mid 5-15 $\mu \mathrm{m}$ region and the continuum due to very small grains does not start to rise until $30 \mu \mathrm{m}$, there is a "trough" in the emission from normal or starburst galaxies (e.g., non-ULIRGS). Then, as the PAH features shift into the $24 \mu \mathrm{m}$ MIPS band at a redshift of $z \simeq 2$, this will lead to a "bump" in the source counts with flux (e.g., distance; Fig. 4; Lagache et al. 2004). These studies demonstrate that PAHs are present at abundances of $\simeq 10 \%$ of the elemental $\mathrm{C}$ in the star forming galaxies as far as Spitzer could probe and that the typical galaxy in these studies 


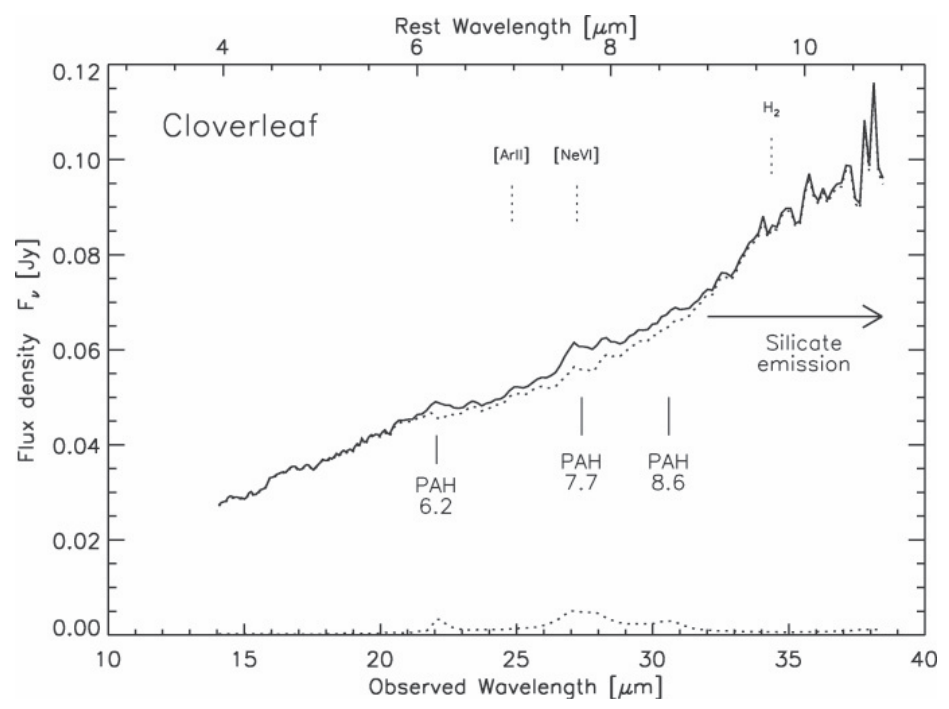

Fig. 3. The infrared spectrum of the Cloverleaf system is characterized by a strong continuum - reminiscent of AGN sources - with distinct PAH features superimposed. The strength of these PAH features indicates a star formation rate of some $10^{3} M_{\odot} / \mathrm{yr}$ (Lutz et al. 2007).
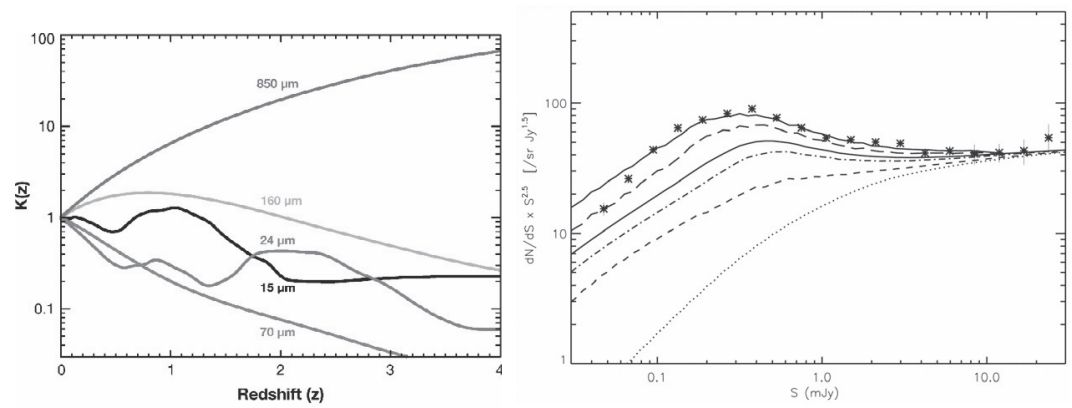

Fig. 4. (a) The K-band correction in different filters shows a "bump" when the PAH emission features shift through the wavelength range covered. (b) Redshift contribution to the number counts at $24 \mu \mathrm{m}$. The dotted, dashed, dash-dotted, tripledot-dashed, and long-dashed lines correspond to the number counts up to redshifts $0.3,0.8,1,1.3$, and 2, respectively. Taken from Lagache et al. (2006) \& (2004).

$\left(L \sim 10^{11}-10^{12} L_{\odot} ;\right.$ star formation rates $\left.20-130 M_{\odot} / \mathrm{yr}\right) ; M \simeq 1010-10^{11} M_{\odot}$ has a mid-IR spectrum dominated by the PAH features. Moreover, the Cosmic InfraRed Background between 24 and $160 \mu \mathrm{m}$ is dominated by these galaxies. 


\section{The future}

With Herschel a new era has arrived and the study of the Universe at far-infrared and sub-millimeter wavelengths has come within reach. First and foremost, Herschel will provide a deeper understanding of the dark and cold or lukewarm local Universe through spectroscopic studies of e.g., [OI], [CII], and CO lines in nearby galactic and extragalactic PAH-bright regions. This will link the mid-IR PAH characteristics in a direct way to the local physical conditions. In addition, spectroscopic studies may reveal far-IR spectral signatures of interstellar PAHs which might provide key information on the individual PAH molecules present in space (see Joblin et al., elsewhere in this volume). Finally, with Herschel, deep source counts at long wavelengths, may reveal the signatures of PAH emission bands as they shift throught the $70 \mu \mathrm{m}$ PACS band at a redshift of $\sim 7$. Indeed, at this point it is fair to expand the PAH hypothesis to its strongest version to read:

- PAH molecules formed in the ejecta of the first generation of stars polluting the Universe with heavy atoms in the form of molecules and dust.

- Ever since that time, PAHs are a key component of the "dust" budget of galaxies.

- Ever since that time, PAH molecules have converted a substantial fraction of the UV-visible radiative stellar energy in galaxies to the mid-IR range.

- Ever since that time, PAH molecules are at the basis of a complex chemistry in the ISM of galaxies.

- Ever since that time, PAHs contribute to many key physical processes that affect the basic structure and evolution of the interstellar medium of galaxies.

In short, PAHs are abundant and important throughout the full star forming era of the Universe.

\section{Summary}

Over the last 25 years, the PAH hypothesis has blossomed thanks to a multitude of observational, experimental, and theoretical studies and the dedicated efforts of many scientists. This conference overviews the current status of the field through the many reviews of key topics related to interstellar PAHs and a cross cut of the active research taking place through the many contributed papers. Overall, the PAH hypothesis has shown to be very robust (or adaptable for the more cynically inclined) and "weathered" the many new observational discoveries and insights that have developed over time. This of course, merely presents a challenge to the younger generation to expand on it so much that the originators might not recognize it anymore or even completely disprove it! 


\section{References}

Allamandola, L.J., \& Tielens, A.G.G.M., Interstellar Dust (Kluwer: Dordrecht)

Allamandola, L.J., Tielens, A.G.G.M., \& Barker, J.R., 1985, ApJ, 290, L25

Allamandola, L.J., Tielens, A.G.G.M., \& Barker, J., 1989, ApJS, 71, 733

Cesarsky, C., Abergel, A., Agnese, P., et al., 1996, A\&A, 315, L32

Crawford, Tielens, A.G.G.M., \& Allamandola, L.J., 1985, ApJ, 293, L45

Duley, W., \& Williams, D.A., 1981, MNRAS, 196, 269

Gillett, F.C., Forest, W.J., \& Merrill, K.M., 1973, ApJ, 183, 87

de Graauw, Th., Haser, L.N., Beintema, D.A., et al., 1996, A\&A, 315, L49

Houck, J., Roellig, T.L., van Cleve, J., et al., 2004, ApJS, 154, 18

Lagache, G., Dole, H., Puget, J.-L., et al., 2004, ApJS, 154, 112

Lagache, G., Puget, J.-L., \& Dole, H, 2006, ARAA, 43, 727

Léger, A., \& d'Hendecourt, L., 1985, A\&A, 146, 81

Léger, A., \& Puget, J.-L., 1984, A\&A, 137, L5

Lepp, S., \& Dalgarno, A., 1988, ApJ, 324, 553

Low, F.J., Young, E., Beintema, D.A., et al., 1984, ApJ, 278, L19

Lutz, D., Yan, L., Armus, L., et al., 2005, ApJ, 632, L13

Lutz, D., Sturm, E., Tacconi, L.J., et al., 2007, ApJ, 661, L25

Olnon, F., Raimond, E., Neugebauer, G., et al., 1986, A\&AS, 65, 607

Peeters, E., Hony, S., Van Kerckhoven, C., et al., 2002, A\&A, 390, 1089

Pope, A., Chary, R.-R., Alexander, D.M., et al., 2008, ApJ, 675, 1171

Puget, J.-L., Léger, A., \& Boulanger, F., 1985, A\&A, 142, L19

Puget, J.-L., \& Léger, A., 1989, ARAA, 27, 161

Rigby, J.R., Marcillac, D., Egami, E., et al., 2008, ApJ, 675, 262

Russel, R., Soifer, B.T., \& Willner, S.P., 1977, ApJ, 217, 149

Sellgren, K., 1984, ApJ, 277, 623

Smith, J.D.T., Draine, B.T., Dale, D.A., et al., 2007, ApJ, 656, 770

Tielens, A.G.G.M., 2008, ARAA, 46, 289

van der Zwet, G., \& Allamandola, L.J., 1985, A\&A, 146, 76

Verstraete, L., Leger, A., D'Hendecourt, L., Defourneau, D., \& Dutuit, O., 1990, A\&A, 237,436

Willner, S.P., Puetter, R.C., Russell, R.W., Soifer, B.T., 1979, ApSS, 65, 95 